Supporting Information

\title{
Charging System Optimization of Triboelectric Nanogenerator for Water Wave Energy Harvesting and Storage
}

Yanyan Yao, ${ }^{a \perp}$ Tao Jiang, ${ }^{a \perp}$ Limin Zhang, ${ }^{a}$ Xiangyu Chen, ${ }^{a}$ Zhenliang Gao, ${ }^{a}$ and Zhong Lin Wang ${ }^{* a b}$

\author{
${ }^{a}$ Beijing Institute of Nanoenergy and Nanosystems, Chinese Academy of Sciences; National Center for \\ Nanoscience and Technology (NCNST), Beijing 100083, China \\ ${ }^{b}$ School of Materials Science and Engineering, Georgia Institute of Technology, Atlanta, GA 30332-0245, \\ USA
}

*E-mail: zlwang@gatech.edu.

${ }^{\perp}$ These authors contribute equally to this work. 


\section{Dynamic output characteristic of TENG under direct water wave impact}

Figure S1 shows the real-time output charge, current, and voltage of wavy-structured TENG connected with a resistor under direct water wave impact at a frequency of $2 \mathrm{~Hz}$. They are all periodic waves, so we only show the results in one cycle. The transferred charge increases rapidly to its peak value at half-cycle and then drops. The current first increases to its peak and then drops to zero at about half-cycle, and in the second half it changes the direction. With increasing the resistance, the current peak decreases, while the voltage shows the opposite trend.
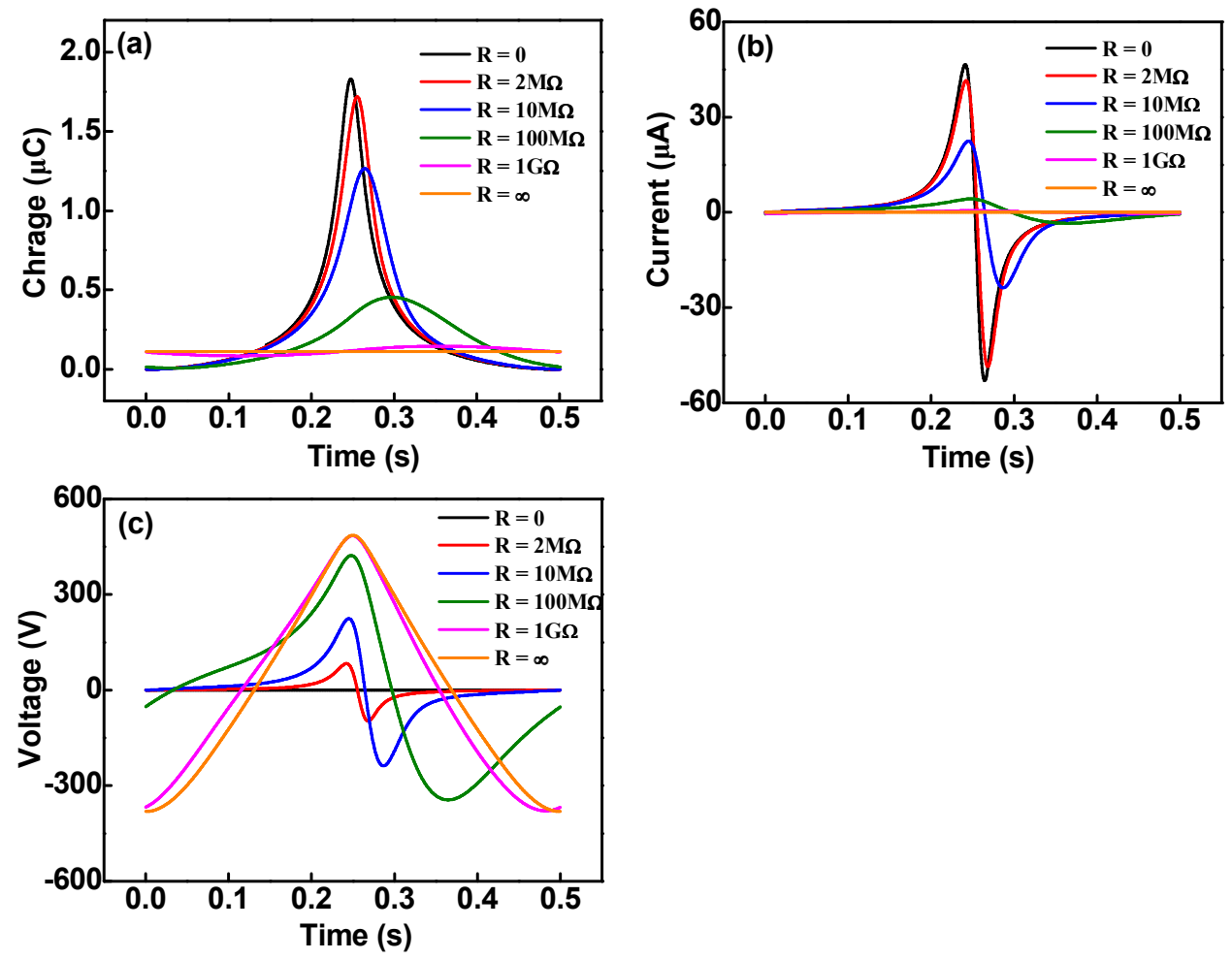

Figure S1. (a) Charge-time relationship, (b) current-time relationship, and (c) voltage-time relationship for the wavy-structured TENG at different load resistances when imposed a periodic triggering at a frequency of $2 \mathrm{~Hz}$.

\section{Formula derivations and calculation results on charging characteristic of TENG under direct water wave impact}

\section{(1) For the first two charging cycles:}

Figure S2 is the simplified diagram of Figure 1c in the first half of each cycle through ideal diode approximation, in which the serial connection of $V_{\mathrm{OC}}$ and $C_{\mathrm{T}}$ is connected with the load capacitor across a 
node $\mathrm{M}$. The charging voltage and stored charge on $C_{\mathrm{L}}$ in the first cycle have been derived in the text. Now we derived the formulae in the second charging cycle. First, according to Equation (6) in the text, we can calculate the voltage and stored charge at the end of first cycle by

$V_{1,2 \text { end }}^{\mathrm{C}}=-\frac{2 C_{\mathrm{L}} Q_{\mathrm{SC}, \text { max }}}{\left(C_{\text {max }}+C_{\mathrm{L}}\right)\left(C_{\text {min }}+C_{\mathrm{L}}\right)}, \quad Q_{1,2 \text { end }}^{\mathrm{C}}=-\frac{2 C_{\mathrm{L}}^{2} Q_{\mathrm{SC}, \text { max }}}{\left(C_{\text {max }}+C_{\mathrm{L}}\right)\left(C_{\text {min }}+C_{\mathrm{L}}\right)}$

The transferred charge on the inherent capacitance $C_{\mathrm{T}}$ is given by

$Q_{1,2 \text { end }}=Q_{1,2 \text { end }}^{\mathrm{C}}-Q_{1, \text { mid }}^{\mathrm{M}}=\frac{2 C_{\mathrm{L}} C_{\text {min }} Q_{\mathrm{SC}, \text { max }}}{\left(C_{\text {max }}+C_{\mathrm{L}}\right)\left(C_{\text {min }}+C_{\mathrm{L}}\right)}$

For the second cycle, in the 1st half, the following equations are satisfied based on the charging principle of TENG

$$
\begin{aligned}
& V_{2,1 \mathrm{st}}^{\mathrm{C}}=-\frac{1}{C_{\mathrm{T}}(t)} \times Q_{2,1 \mathrm{st}}+V_{\mathrm{OC}}(t)=\frac{Q_{2,1 \mathrm{st}}^{\mathrm{C}}}{C_{\mathrm{L}}}, \quad Q_{2,1 \mathrm{st}}^{\mathrm{C}}-Q_{2,1 \mathrm{st}}=Q_{2}^{\mathrm{M}} \\
& V_{2,1 \mathrm{st}}^{\mathrm{C}}=\frac{Q_{\mathrm{SC}}(t)+Q_{2}^{\mathrm{M}}}{C_{\mathrm{T}}(t)+C_{\mathrm{L}}}, \quad Q_{2,1 \mathrm{st}}^{\mathrm{C}}=\frac{Q_{\mathrm{SC}}(t)+Q_{2}^{\mathrm{M}}}{C_{\mathrm{T}}(t)+C_{\mathrm{L}}} C_{\mathrm{L}}
\end{aligned}
$$

where the stored charges $Q_{2}^{\mathrm{M}}$ on node $\mathrm{M}$ at the beginning of the second cycle can be obtained by

$$
Q_{2}^{\mathrm{M}}=-Q_{1,2 \text { end }}-Q_{1,2 \text { end }}^{\mathrm{C}}=\frac{2 C_{\mathrm{L}} Q_{\mathrm{SC}, \text { max }}\left(C_{\mathrm{L}}-C_{\text {min }}\right)}{\left(C_{\text {max }}+C_{\mathrm{L}}\right)\left(C_{\text {min }}+C_{\mathrm{L}}\right)}
$$

By combining Equations (S4) and (S5), we can calculate the charging voltage and stored charge in the 1st half of the second cycle. Then the charges stored on node $\mathrm{M}$ at the beginning of the 2 nd half of the second cycle $\left(Q_{2, \text { mid }}^{\mathrm{M}}\right)$ can be given by

$$
Q_{2, \text { mid }}^{\mathrm{M}}=-Q_{2,2 \text { begin }}+Q_{2,2 \text { begin }}^{\mathrm{C}}=-Q_{2, \text { lend }}-Q_{2, \text { lend }}^{\mathrm{C}}=-\frac{2 C_{\mathrm{L}} Q_{\mathrm{SC}, \text { max }}+\left(C_{\mathrm{L}}-C_{\text {max }}\right) Q_{2}^{\mathrm{M}}}{C_{\text {max }}+C_{\mathrm{L}}}
$$

In the 2nd half of the second cycle, we easily obtained

$$
V_{2,2 \mathrm{nd}}^{\mathrm{C}}=-\frac{Q_{2,2 \mathrm{nd}}}{C_{\mathrm{T}}(t)}+V_{\mathrm{OC}}(t)=\frac{Q_{2,2 \mathrm{nd}}^{\mathrm{C}}}{C_{\mathrm{L}}}, \quad Q_{2,2 \mathrm{nd}}^{\mathrm{C}}-Q_{2,2 \mathrm{nd}}=Q_{2, \text { mid }}^{\mathrm{M}}
$$

Finally, we derived out the formula for $V_{2,2 n d}^{\mathrm{C}}$ as follows

$$
V_{2,2 \text { nd }}^{\mathrm{C}}=\frac{Q_{\mathrm{SC}}(t)+Q_{2, \text { mid }}^{\mathrm{M}}}{C_{\mathrm{T}}(t)+C_{\mathrm{L}}}=\frac{Q_{\mathrm{SC}}(t)-\frac{4 C_{\mathrm{L}} Q_{\mathrm{SC}, \text { max }}\left(C_{\mathrm{L}}^{2}+C_{\text {max }} C_{\text {min }}\right)}{\left(C_{\max }+C_{\mathrm{L}}\right)^{2}\left(C_{\min }+C_{L}\right)}}{C_{\mathrm{T}}(t)+C_{\mathrm{L}}}
$$


Based on the above derivations, the voltage profiles of $V_{k}^{\mathrm{C}}$ and $\left|V_{k}^{\mathrm{C}}\right|$ in the first two cycles are shown in Figure S3, which can reflect the charging characteristic under the direct wave impact.

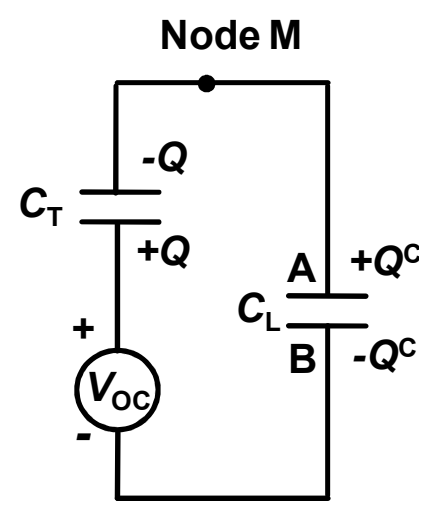

Figure S2. Simplified circuit diagram of the charging system in the first half of each cycle under direct water wave impact.
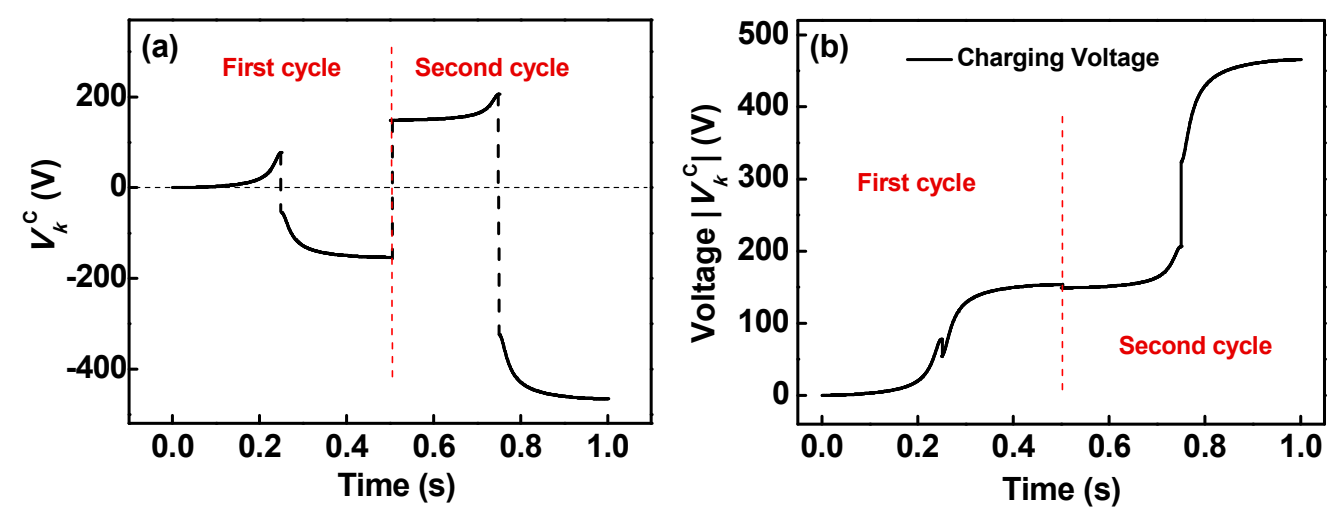

Figure S3. Profiles of (a) voltage $V_{k}^{\mathrm{C}}$ and (b) voltage $\left|V_{k}^{\mathrm{C}}\right|$ in the first two cycles for the charging system with $C_{\mathrm{L}}=5 \mathrm{nF}$ under direct water wave impact.

\section{(2) For the $k$-th charging cycle:}

Similar to the previous derivations in the first two cycles, in the 1 st half of the $k$-th charging cycle, the charging voltage and charge stored on the $C_{\mathrm{L}}$ can be given by

$V_{k, 1 \mathrm{st}}^{\mathrm{C}}=\frac{Q_{\mathrm{SC}}(t)+Q_{k}^{\mathrm{M}}}{C_{\mathrm{T}}(t)+C_{\mathrm{L}}}, \quad Q_{k, 1 \mathrm{st}}^{\mathrm{C}}=\frac{Q_{\mathrm{SC}}(t)+Q_{k}^{\mathrm{M}}}{C_{\mathrm{T}}(t)+C_{\mathrm{L}}} C_{\mathrm{L}}$

Therefore, at the end of this half, the voltage and charges are given by 
$V_{k, \text { lend }}^{\mathrm{C}}=\frac{Q_{\mathrm{SC}, \text { max }}+Q_{k}^{\mathrm{M}}}{C_{\text {max }}+C_{\mathrm{L}}}, \quad Q_{k, \text { lend }}^{\mathrm{C}}=\frac{Q_{\mathrm{SC}, \text { max }}+Q_{k}^{\mathrm{M}}}{C_{\text {max }}+C_{\mathrm{L}}} C_{\mathrm{L}}, \quad Q_{k, \text { lend }}=\frac{C_{\mathrm{L}} Q_{\mathrm{SC}, \text { max }}-C_{\text {max }} Q_{k}^{\mathrm{M}}}{C_{\text {max }}+C_{\mathrm{L}}}$

Then the charges stored on Node M at the beginning of the 2 nd half of the $k$-th cycle can be obtained by

$Q_{k, \text { mid }}^{\mathrm{M}}=-Q_{k, 2 \text { begin }}+Q_{k, 2 \text { begin }}^{\mathrm{C}}=-Q_{k, \text { lend }}-Q_{k, \text { lend }}^{\mathrm{C}}$

At the end of this half, the charges stored on the $C_{\mathrm{T}}$ and $C_{\mathrm{L}}$ are given by

$Q_{k, \text { end }}=\frac{-Q_{k, \text { mid }}^{\mathrm{M}} C_{\text {min }}}{C_{\text {min }}+C_{\mathrm{L}}}, \quad Q_{k, 2 \text { end }}^{\mathrm{C}}=\frac{Q_{k, \text { mid }}^{\mathrm{M}} C_{\mathrm{L}}}{C_{\text {min }}+C_{\mathrm{L}}}$

For the $(k+1)$-th cycle, the charges stored on Node $\mathrm{M}$ at the beginning of this cycle can be given by

$Q_{k+1}^{\mathrm{M}}=-Q_{k+1,1 \text { begin }}+Q_{k+1, \text { begin }}^{\mathrm{C}}=-Q_{k, 2 \text { end }}-Q_{k, \text { end }}^{\mathrm{C}}=\frac{Q_{k, \text { mid }}^{\mathrm{M}}\left(C_{\text {min }}-C_{\mathrm{L}}\right)}{C_{\text {min }}+C_{\mathrm{L}}}$

A recursion relationship for $Q_{k}^{\mathrm{M}}$ can be concluded from Equations (10)-(13)

$Q_{k+1}^{\mathrm{M}}=\frac{C_{\mathrm{L}}-C_{\max }}{C_{\mathrm{L}}+C_{\text {max }}} \frac{C_{\mathrm{L}}-C_{\text {min }}}{C_{\mathrm{L}}+C_{\text {min }}} Q_{k}^{\mathrm{M}}+2 \frac{C_{\mathrm{L}}-C_{\text {max }}}{C_{\mathrm{L}}+C_{\text {max }}} \frac{C_{\mathrm{L}}}{C_{\mathrm{L}}+C_{\text {min }}} Q_{\mathrm{SC}, \max }$

By applying the initial boundary condition of $Q_{1}^{\mathrm{M}}=0$, Equation (S14) can be solved as

$Q_{k}^{\mathrm{M}}=\frac{C_{\mathrm{L}}-C_{\text {min }}}{C_{\text {max }}+C_{\text {min }}} Q_{\mathrm{SC}, \text { max }}-\frac{C_{\mathrm{L}}-C_{\text {min }}}{C_{\text {max }}+C_{\text {min }}} Q_{\mathrm{SC}, \text { max }} \times\left[\frac{\left(C_{\mathrm{L}}-C_{\max }\right)\left(C_{\mathrm{L}}-C_{\min }\right)}{\left(C_{\mathrm{L}}+C_{\max }\right)\left(C_{\mathrm{L}}+C_{\min }\right)}\right]^{k-1}$

which is the same as Equation (7) in the text. Then the voltage and stored energy on $C_{\mathrm{L}}$ at the end of $k$-th charging cycle can be calculated.

Figure $\mathrm{S} 4$ shows the stored power at different load capacitance $C_{\mathrm{L}}$ at $x_{\max }=6 \mathrm{~mm}$ and we can see the charging time to get the maximum stored power is smaller for smaller $C_{\mathrm{L}}$ and the maximum stored power is independent of the load capacitance $C_{\mathrm{L}}$.

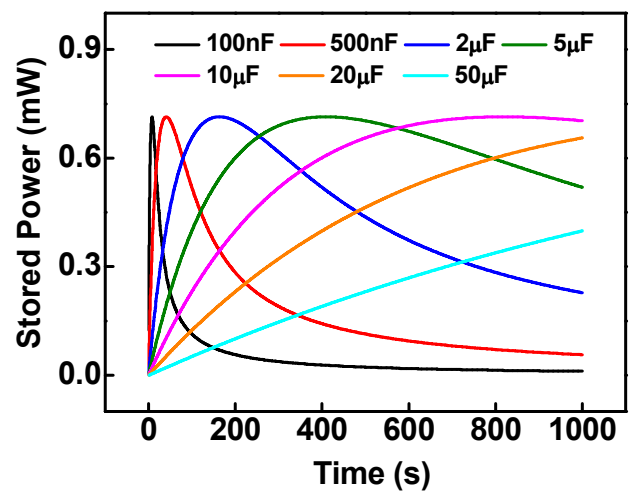

Figure S4. Stored power-time relationships at different $C_{\mathrm{L}}$ when $x_{\max }=6 \mathrm{~mm}$. 


\section{Experimental details for the real water wave tests}

Fabrication of wavy-structured TENG. First, a $125 \mu \mathrm{m}$ thick Kapton film was periodically bent into a wavy shape by using a set of glass rods with diameter of $6 \mathrm{~mm}$. The set was sent into a furnace and baked at $360{ }^{\circ} \mathrm{C}$ for $2 \mathrm{~h}$. Because the Kapton film is thermoplastic, it can remain in the wavy shape stably below its glass transition temperature. Then $200 \mathrm{~nm}$ thick copper was sputtered on both sides of the wavy Kapton film as electrodes. Second, two $20 \mu \mathrm{m}$ thick FEP films (purchased from DuPont Company) cleaned by the alcohol, acetone and deionized water in sequence, were deposited with a layer of copper on one side as the electrode. The copper sides of two FEP-Cu films were tightly attached to two acrylic substrates with a size of $8 \mathrm{~cm} \times 8$ $\mathrm{cm}$. By placing a needle above the FEP surface of FEP-Cu film with a height of $1 \mathrm{~cm}$, connecting the needle to the cathode, and connecting the $\mathrm{Cu}$ electrode to the anode and ground, electrons were injected to the top surface of FEP film. A polarization voltage of $5 \mathrm{kV}$ was applied for $5 \mathrm{~min}$. Third, by sandwiching the wavy $\mathrm{Cu}-\mathrm{Kapton}-\mathrm{Cu}$ film using two acrylic substrates with FEP films facing inside and bonding the structures together with adhesive tape, the wavy-structured TENG device was finally obtained.

For the water wave tests, four wavy-structured TENG units were vertically anchored onto the internal surface of acrylic plates in the center position of an acrylic box. The four TENGs were connected to a rectifier bridge and then connected in parallel. A steel ball with the diameter of $3.5 \mathrm{~cm}$ was placed in the center of the device, and then the waterproofing was processed. The sealed device was used to charge various capacitors. The charging voltages on various load capacitances $C_{\mathrm{L}}$ under the enclosed ball collision were measured by a voltage-meter (Keithley 6514 System Electrometer). 